\title{
A Novel Method for Intraoperative Reduction of the Mobile Meniscal Bearing in the Oxford Unicompartmental Knee Replacement
}

\author{
Simon M. Thompson, Daniel G. G. Wilson, Nimesh Patel, and Neil W. Bradley \\ The Royal Surrey County Hospital, Egerton Road, Guildford, Surrey GU2 7XX, England \\ Address correspondence to Simon M. Thompson,s.m.thompson96@imperial.ac.uk
}

Received 16 July 2014; Revised 16 December 2014; Accepted 24 February 2015

Copyright (C) 2015 Simon M. Thompson et al. This is an open access article distributed under the terms of the Creative Commons Attribution License, which permits unrestricted use, distribution, and reproduction in any medium, provided the original work is properly cited.

\begin{abstract}
Insertion of the bearing can be challenging due to patient body habitus or limited exposure when using a minimally invasive technique. We describe a novel way of inserting the bearing not previously described in the literature. The bearing is positioned on the femoral component in full flexion. Then, with a gentle extension of the knee, the bearing snaps into position and is seated correctly on a consistent basis.
\end{abstract}

Keywords reduction; mobile meniscal bearing; Oxford unicompartmental knee replacement

\section{Background}

Unicompartmental knee replacement (UKR) was first developed in the 1950s by McKeever and MacIntosh. In 1978, Goodfellow [1] introduced the meniscal bearing forming the basis of what is now known as the Oxford Partial Knee. Insertion of the bearing can be challenging due to patient body habitus or limited exposure when using a minimally invasive technique. We describe a novel way of inserting the bearing. A feeler gauge is used to ensure the thickness of the desired bearing and that the flexion and extension gaps are equal, to avoid damage to the bearing or medial femoral condyle.

\section{Method}

1. Initial placement of the bearing on the femoral component in full flexion (Figure 1).

2. Gently extend the knee (Figure 2).

3. Bearing snaps into position and is seated correctly (Figure 3).

\section{Discussion}

UKR is a technically demanding procedure especially when performed through a minimally invasive approach. This can affect outcome $[2,3]$. The mobile bearings may also dislocate, and rates have been quoted as being between $0.5 \%$ and $10 \%[4,5]$. Therefore the bearing cannot afford to be undersized.

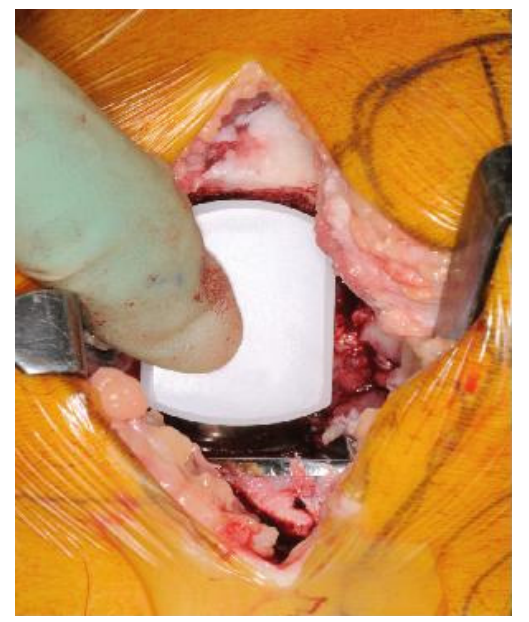

Figure 1: Initial placement of the bearing on the femoral component in full flexion.

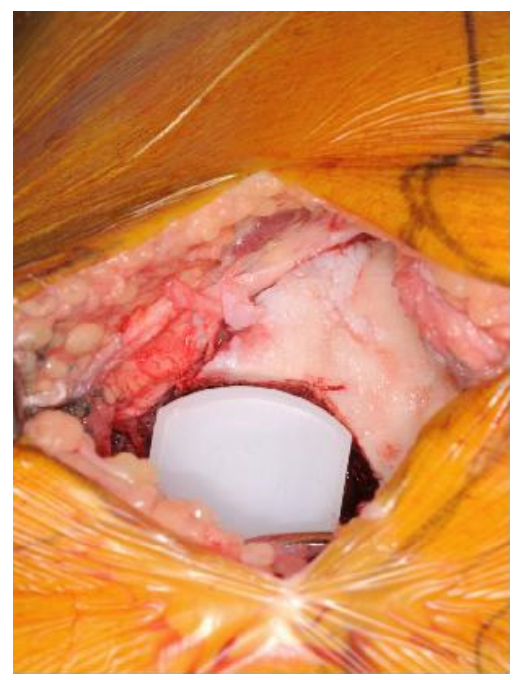

Figure 2: Gentle extension of the knee. 


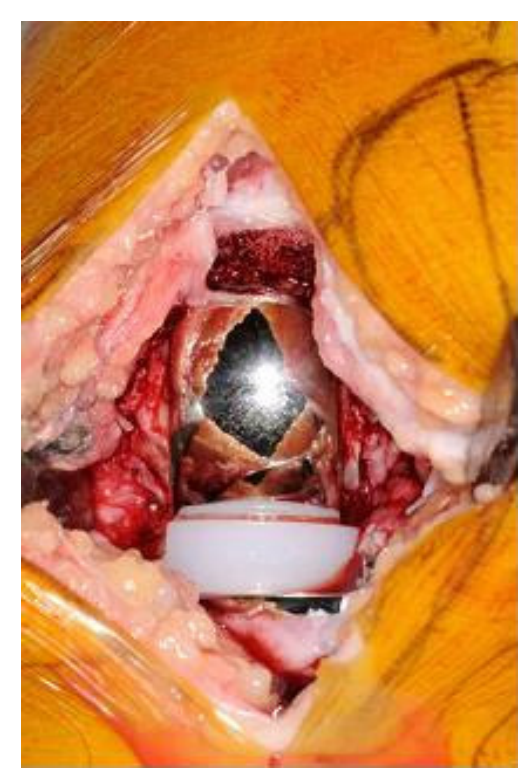

Figure 3: Bearing snaps into position and is seated correctly.

Surgical technique from Biomet states that "the reconstruction is completed by snapping the chosen bearing into place". This is usually achieved with the knee in flexion and placing the bearing into the flexion gap created intraoperatively. This process, whilst normally straightforward, can be difficult. This technique is reproducible and is a useful aid for tough reductions.

\section{References}

[1] J. Goodfellow and J. O'Connor, The mechanics of the knee and prosthesis design, J Bone Joint Surg Br, 60-B (1978), 358-369.

[2] E. Koskinen, A. Eskelinen, P. Paavolainen, P. Pulkkinen, and V. Remes, Comparison of survival and cost-effectiveness between unicondylar arthroplasty and total knee arthroplasty in patients with primary osteoarthritis: a follow-up study of 50,493 knee replacements from the Finnish Arthroplasty Register, Acta Orthop, 79 (2008), 499-507.

[3] O. Furnes, B. Espehaug, S. A. Lie, S. E. Vollset, L. B. Engesaeter, and L. I. Havelin, Failure mechanisms after unicompartmental and tricompartmental primary knee replacement with cement, The Journal of bone and joint surgery American volume, 89 (2007), $519-525$.

[4] D. W. Murray, J. W. Goodfellow, and J. J. O'Connor, The Oxford medial unicompartmental arthroplasty: A ten-year survival study, J Bone Joint Surg Br, 80 (1998), 983-989.

[5] H. Pandit, C. Jenkins, D. J. Beard, A. J. Price, H. S. Gill, C. A. Dodd, et al., Mobile bearing dislocation in lateral unicompartmental knee replacement, Knee, 17 (2010), 392-397. 